\title{
Real-time, step-wise, electrical detection of protein molecules using dielectrophoretically aligned SWNT-film FET aptasensors
}

\author{
Taechang An, ${ }^{a}$ Ki Su Kim, ${ }^{b}$ Sei Kwang Hahn ${ }^{* b}$ and Geunbae Lim*ac
}

Received 30th April 2010, Accepted 18th June 2010

DOI: $10.1039 / \mathbf{c 0 0 5 2 7 6 k}$

\begin{abstract}
Aptamer functionalized addressable SWNT-film arrays between cantilever electrodes were successfully developed for biosensor applications. Dielectrophoretically aligned SWNT suspended films made possible highly specific and rapid detection of target proteins with a large binding surface area. Thrombin aptamer immobilized SWNT-film FET biosensor resulted in a real-time, label-free, and electrical detection of thrombin molecules down to a concentration of ca. $7 \mathrm{pM}$ with a step-wise rapid response time of several seconds.
\end{abstract}

Aptamers are oligonucleotides which can specifically bind to target molecules such as small molecules, peptides, proteins, cells, and other biomolecules. Aptamers can be prepared by systematic evolution of ligands by exponential enrichment (SELEX) process. ${ }^{1-3}$ Owing to the high binding affinity and selectivity to target biomolecules, aptamers have been used for various biosensor applications. ${ }^{4-7}$ Aptasensor is the term for biosensors using aptamers for the detection of biomolecules. With the development of nanotechnology, a real-time, labelfree, and electrical detection of biomolecules was successfully carried out with the aptasensors. They have been developed using various nano-materials such as single-walled carbon nanotube (SWNT), ${ }^{8}$ conducting polymers, ${ }^{9}$ graphenes, ${ }^{10}$ and silicone nanowires (Si$\mathrm{NW})^{11,12}$ for real-time electrical detection of biomolecules. However, the aptasensors still had several problems such as difficulties in fabrication and spatial control, reproducibility, a long detection time, insignificant electrical signals, and an unproportional sensitivity to the concentration of target molecules. To circumvent these technical hurdles, SWNT has been widely investigated for various biosensor applications.

SWNT-based biosensors have been initially developed using individual nanotubes ${ }^{13-15}$ resulting in many problems described above. These problems could be alleviated using SWNT-film based biosensors for the detection of DNA, ${ }^{16}$ proteins, ${ }^{17}$ and bacteria. ${ }^{18}$ In particular, it was made possible to minimize device variation, and generate a large active area and a high current output with a relative insensitivity to the spatial position. ${ }^{19}$ SWNT-film based biosensors resulted in an improved signal-to-noise ratio and an enhanced detection limit in comparison to the individual nanotube systems. ${ }^{20}$ Furthermore, the sensitivity of SWNT-film based biosensors could be

${ }^{a}$ Department of Mechanical Engineering, Pohang University of Science and Technology (POSTECH), San 31, Hyoja-dong, Nam-gu, Pohang, Kyungbuk,790-784, Korea.E-mail: limmems@postech.ac.kr

${ }^{b}$ Department of Material Science and Engineering, Pohang University of Science and Technology (POSTECH), San 31, Hyoja-dong, Nam-gu, Pohang, Kyungbuk, 790-784, Korea. E-mail: skhanb@postech.ac.kr

'Department of Integrative Bioscience and Biotechnology, Pohang University of Science and Technology (POSTECH), San 31, Hyojadong, Nam-gu, Pohang, Kyungbuk, 790-784, Korea improved by suspending the SWNT-film away from the floor providing more than twice the binding surface area with increased convectional and diffusional fluxes. ${ }^{21}$ In this work, we developed a new one-step method for the fabrication of dielectrophoretically aligned addressable SWNT-films and demonstrated a real-time, stepwise, rapid, electrical detection of thrombin molecules using the SWNT-film FET aptasensors.

Fig. 1 shows a schematic representation for the fabrication of thrombin aptamer functionalized SWNT-film FET biosensors. Cantilever electrode arrays were fabricated by a general MEMS process. A low stress silicon nitride $(1 \mu \mathrm{m})$ was deposited on a $\mathrm{Si}$ wafer using a low pressure chemical vapor deposition (LPCVD) for insulation and a cantilever structure. Gold electrode arrays $(\mathrm{Cr} / \mathrm{Au}$ 20/200 nm) were patterned on the SiN surface through a lift-off process. Then, cantilever arrays were patterned by $\mathrm{SiN}$ reactive ion etching (RIE). The Si below the cantilever electrodes was etched by isotropic wet etching to form a suspended cantilever structure. Finally, SU8 was coated on the electrodes for passivation except for the cantilever surface exposing the SWNT-films to target molecules (Fig. 1a). On the other hand, a suspended SWNT-COOH film was prepared by dielectrophoresis and the surface tension of water meniscus. The suspension was placed between the patterned cantilever electrodes. When an AC voltage of $1 \mathrm{MHz}$ frequency and $5 \mathrm{~V}_{\mathrm{p}-\mathrm{p}}$ amplitude was applied, SWNTs were aligned between cantilever electrodes by dielectrophoretic force. The suspension was then partially removed and the remaining suspension formed a water meniscus between the electrodes. The collected SWNTs were compressed by the surface tension of water meniscus and attached to the electrodes by Van der Waals force, forming a suspended SWNTfilm between the electrodes (Fig. 1b). The SWNT density in the film was $c a$. $4 \sim 6$ bundles per $\mu \mathrm{m}^{2}$ having $c a$. 10 SWNTs in each bundle, which could be controlled by changing the amplitude and frequency of AC electric field, ${ }^{22}$ the concentration of SWNT suspension, and the collection time. ${ }^{23}$ The suspended SWNT-films were addressed precisely to electrodes, since they were attached only to the electrodes where an electric field was applied. In addition, the fabrication of multi-functional SWNT-film arrays was made possible by the sitespecific positioning of SWNT-films with same and different compositions on the same chip. It took less than $10 \mathrm{~min}$ to prepare the SWNT-film and $2 \mathrm{~h}$ to conjugate aptamers to the SWNT-film. Moreover, the aptasensor could be exploited repeatedly for the detection of thrombin.

Fig. 2a shows a SEM image of suspended SWNT-film arrays between electrodes. The SWNT-films constructed with several SWNT bundle layers were well aligned and suspended between two cantilever electrodes (Fig. 2b). The large contact area between SWNT-films and the electrodes provided a low contact resistance, a robust mechanical contact for long-term stability, and a large binding region for the biosensor by Schottky barrier modulation 
(a)

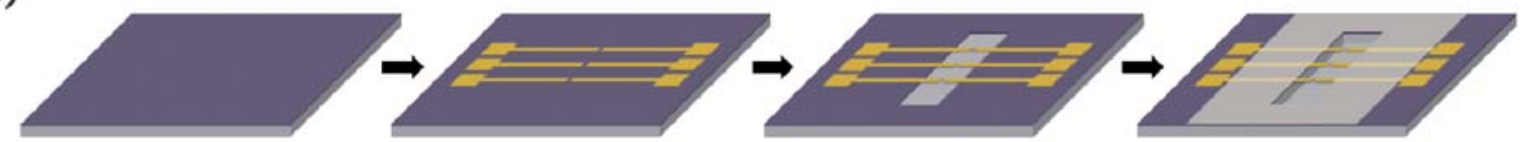

$\mathrm{SiN}$ deposition on Si substrate Gold electrodes patterning

SiN cantilevers patterning Si etching and electrodes passivation

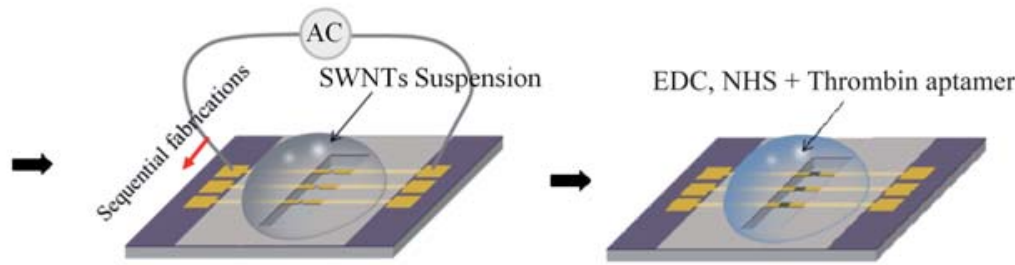

Suspended SWNT film fabrication

Thrombin aptamer immobilization

(b)

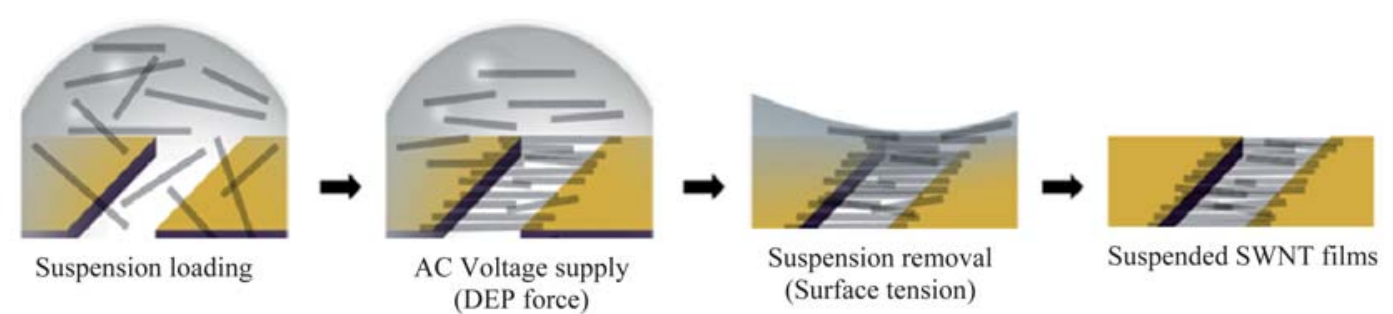

(c)

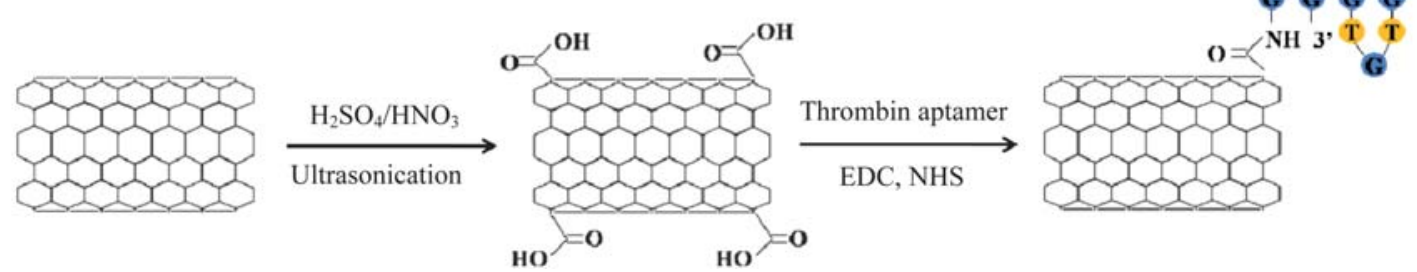

Fig. 1 Schematic representations for (a) the fabrication of thrombin aptamer functionalized SWNT-film field effect transistor (FET) biosensors, (b) the preparation of suspended SWNT-films by dielectrophoresis and surface tension, and (c) the surface modification of SWNT in a strong acid to introduce carboxyl groups and the following covalent immobilization of thrombin aptamers by amide bond formation using 1-ethyl-3-[3-(dimethylamino)propyl]carbodiimide (EDC) and N-hydroxysulfosuccinimide (sulfo-NHS).

between SWNT and the contact electrodes. ${ }^{24}$ The geometrical dimension of SWNT-films was controlled by the width and gap of cantilever electrodes (Fig. 2c). We used cantilever electrodes with a width of $20 \mu \mathrm{m}$ and a gap of $4 \mu \mathrm{m}$. After that, thrombin DNA aptamers were covalently immobilized on suspended SWNT-films for specific binding tests to target proteins. Fig. 1c shows a schematic for the surface modification of SWNT with thrombin aptamers. SWNT-COOH was prepared by the oxidation of carbon nanotubes in a strong acid solution with sonication. ${ }^{25}$ SWNT $(5 \mathrm{mg})$ was suspended in $50 \mathrm{~mL}$ of concentrated $\mathrm{H}_{2} \mathrm{SO}_{4} / \mathrm{HNO}_{3}(3 / 1$, v/v) and sonicated in an ultrasonication bath for $3 \mathrm{~h}$. The nanotube suspension was diluted with DI water and then centrifuged at $12000 \mathrm{rpm}$ for $10 \mathrm{~min}$. The remaining acid was completely removed by decanting the supernatant and washing with DI water, repeatedly. When the $\mathrm{pH}$ of the suspension was 7.4, the carbon nanotubes were re-dispersed in DI water by ultrasonication. Thrombin DNA aptamers were immobilized as described elsewhere. ${ }^{12}$ Briefly, thrombin aptamer with an amine terminal group of $5^{\prime}-\mathrm{NH}_{2}-\mathrm{d}$ (GGTTGGTGTGGTTGG)- $3^{\prime}$ was synthesized by using an oligonucleotide synthesizer. Thrombin aptamers were dissolved in phosphate buffer ( $\mathrm{PB}, 200 \mathrm{mmol}, \mathrm{pH} 8)$ to prepare an aptamer solution at a concentration of $20 \mu \mathrm{mol}$. For covalent surface immobilization, the SWNT-COOH films were treated with $10 \mathrm{~mL}$ of $\mathrm{PB}$ and surface-activated by the addition of 1-ethyl-3-[3-(dimethylamino)propyl]carbodiimide (EDC, $3.0 \mathrm{mg}$ ) and $\mathrm{N}$-hydroxysulfosuccinimide (sulfo-NHS, $1.7 \mathrm{mg}$ ). Then, the aptamer solution was added and incubated at room temperature for amide bond formation between the amine group of the aptamer and the carboxyl group of SWNT-COOH film. The resulting aptasensor 

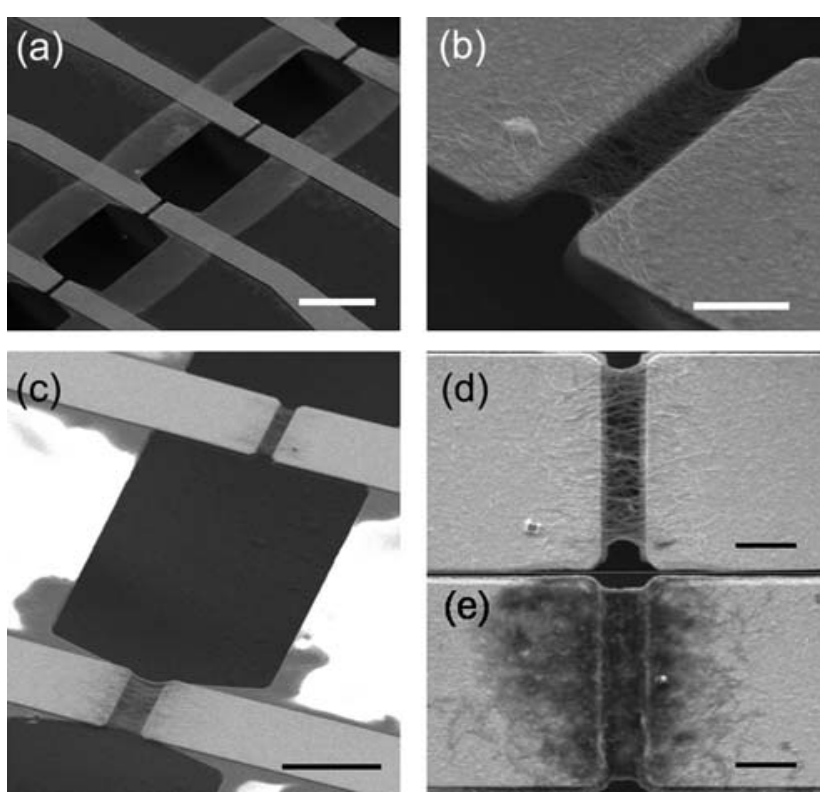

Fig. 2 SEM images of suspended SWNT-film arrays at the magnifications of (a) $\times 400$ (scale bar: $50 \mu \mathrm{m}$ ) and (b) $\times 5,000$ (scale bar: $5 \mu \mathrm{m}$ ). (c) SEM image of suspended SWNT-films with a different channel length (scale bar: $20 \mu \mathrm{m}$ ). SEM images of aptamer functionalized SWNT-films (d) before and (e) after thrombin binding (scale bars: $5 \mu \mathrm{m}$ ).

was rinsed extensively with PB to remove the remaining EDC and sulfo-NHS. ${ }^{25}$ In order to prevent the nonspecific binding, Tween 20 was coated on the unoccupied sites of SWNT. ${ }^{26}$ As shown in Fig. 2b and $2 \mathrm{~d}$, the thickness of SWNT-film increased after the covalent immobilization of aptamers.

The electrical properties of the SWNT-films were analyzed in real time at room temperature using a precision semiconductor parameter analyzer (HP 4156A). Fig. 3 shows the $I-V$ characteristics of suspended SWNT-films in a buffer solution at $\mathrm{pH}$ 5.6. The source-drain current decreased when the electrolyte gate bias increased by the reference electrode. In other words, the suspended SWNT-films exhibited p-type characteristics in the buffer solution. ${ }^{22}$ For a real time electrical detection of protein, nonspecific and specific binding

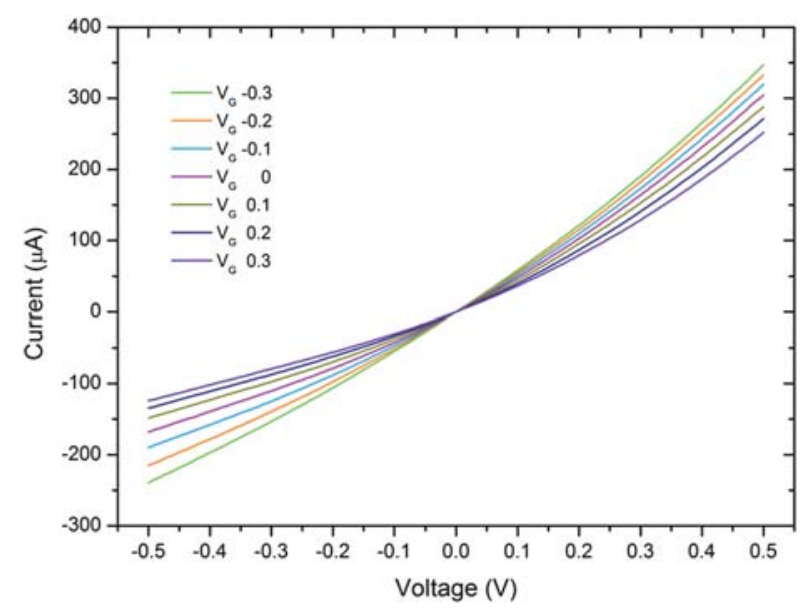

Fig. 3 Electrical characteristics of suspended SWNT-films at various electrolyte gate voltages from $-0.3 \mathrm{~V}$ to $0.3 \mathrm{~V}$. tests were carried out using SWNT-COOH films without and with aptamers. Thrombin and VEGF were used as model proteins for the binding tests. In the case of nonspecific binding tests as a control experiment, a $10 \mu \mathrm{L}$ drop of the buffer and protein solutions at $\mathrm{pH}$ 5.6 and specified concentrations were placed on the suspended SWNT-films, and then the conductance of the suspended SWNTfilms was monitored with a $10 \mathrm{mV}$ bias voltage. Fig. $4 \mathrm{a}$ and $4 \mathrm{~b}$ show the current change with increasing concentrations of thrombin and VEGF, respectively. The aptasensor showed a meaningful current change at a concentration of $70 \mathrm{pM}$ with a response time less than 10 s. In addition, the conductance decreased proportionally upon stepwise exposure to both thrombin and VEGF solution at concentrations from $70 \mathrm{pM}$ to $700 \mathrm{nM}$. Proteins can bind to the surface of SWNT-COOH film nonspecifically by electrostatic and hydrophobic interactions, and $\pi-\pi$ stacking between SWNTs. ${ }^{24}$ In order for specific binding tests, thrombin aptamer-functionalized SWNT-film biosensor was surface modified with Tween 20 and assessed in the same manner with the nonspecific binding tests. Each $3 \mu \mathrm{L}$ of the buffer and protein solutions with specified concentrations was added sequentially to the aptasensor using a micropipette. As shown in Fig. 4c, electric current dropped rapidly in a step-wise manner after the injection of thrombin, whereas no current change was observed for the case of VEGF addition. The results confirmed the specific binding of thrombin molecules to the SWNT-film aptasensor. As reported elsewhere, ${ }^{24}$ the treatment with Tween 20 effectively minimized the non-specific binding. Finally, the sensitivity of SWNT-film thrombin aptasensors was investigated with increasing thrombin concentrations (Fig. 4d). The current dropped sharply in a step-wise manner when thrombin samples were added sequentially at the concentrations from $7 \mathrm{pM}$ to $70 \mathrm{nM}$ to the buffer solution. The standard deviation of the noise signal was estimated to be $c a$. 0.059 . The signal detected at the concentration of $7 \mathrm{pM}$ was $c a$. 0.24 , which was four times higher than the noise level and regarded as a significantly meaningful change. The variation of current after the addition of thrombin molecules can be explained by the electrostatic gating effect by the positive charge of thrombin molecules in the buffer at $\mathrm{pH}$ 5.6. In addition, the modulated Schottky barrier between electrodes and dielectrophoretically aligned SWNTs in the SWNT-film decreased the local work function, contributing for an extraordinary rapid response time of several seconds. ${ }^{24}$ As shown in Fig. 2e, the SWNT-film surface was completely covered by thrombin molecules after the binding tests.

In direct comparison with previous reports, ${ }^{10,24,26,27}$ the dielectrophoretically aligned SWNT-film based aptasensor made possible a real-time, step-wise, and electrical detection of proteins with a rapid response time and a high sensitivity proportional to the protein concentration. The response time of nanotube sensors placed on the floor of the substrate was reported to be in the range of tens of seconds to a few min. ${ }^{8,17,26-28}$ In contrast, the dielectrophoretically aligned SWNT-film based aptasensor had a very short response time of a few seconds. In addition, the dielectrophoretically aligned SWNT-film based aptasensor had a lower detection limit of $c a .7 \mathrm{pM}$ than that of the single SWNT biosensors placed on the floor of the substrate (about $10 \mathrm{nM}$ ). ${ }^{8}$ Finally, the SWNT-film based aptasensor provided a proportional sensitivity to the concentration of proteins. Most of the previous SWNT sensors did not exhibit a proportional sensitivity, mostly converging with increasing protein concentrations. ${ }^{8,17,26}$ These patterns might be difficult to be applied in real systems. As shown in the inset of Fig. 4d, however, the sensitivity 
(a)

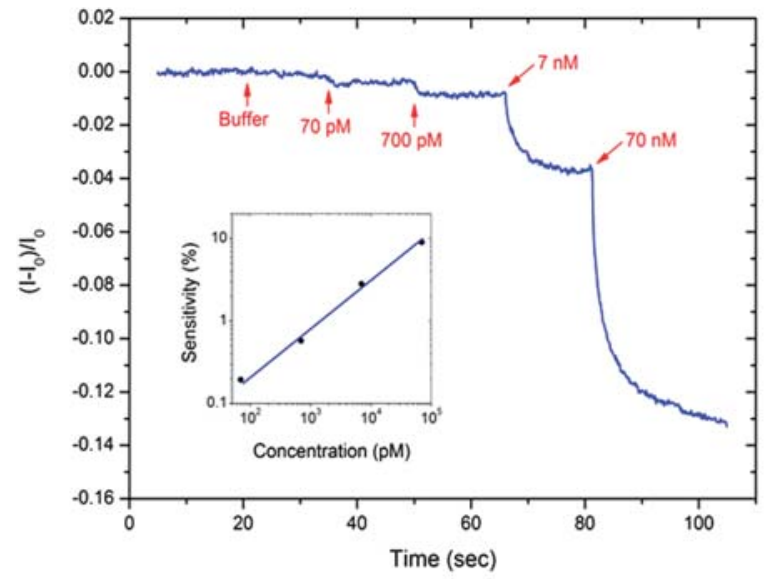

(c)

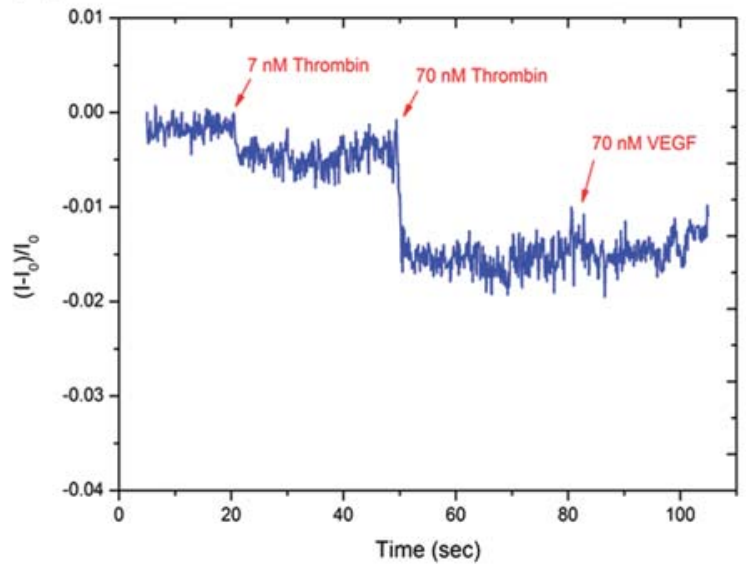

(b)

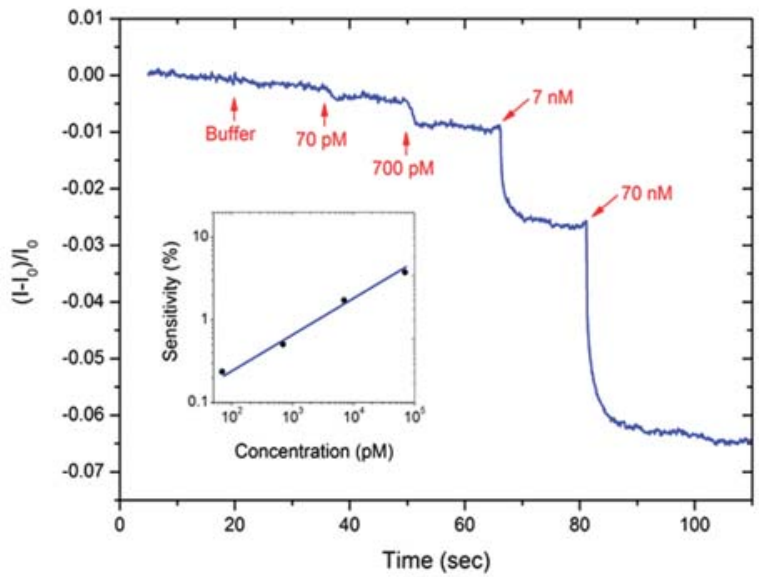

(d)

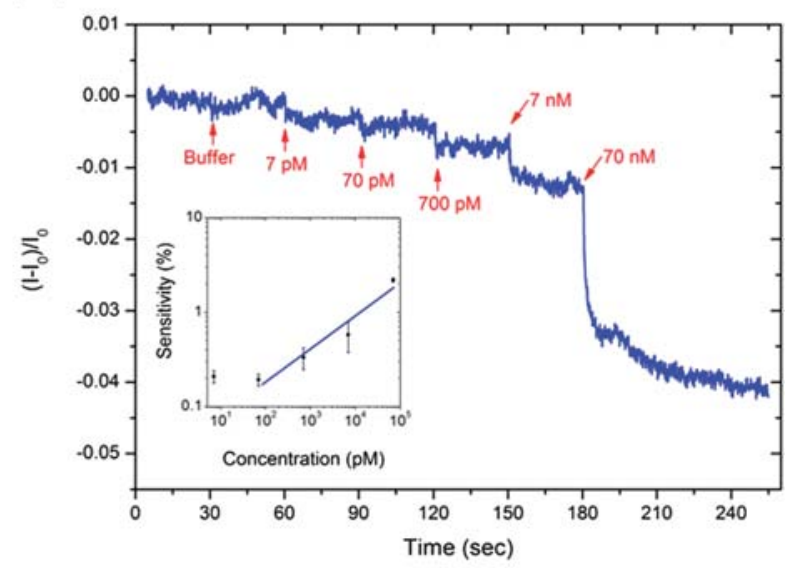

Fig. 4 Real-time electrical detection of proteins using thrombin DNA aptamer-functionalized SWNT-film FET biosensors: Real-time electrical detection for the nonspecific binding of (a) thrombin and (b) VEGF to the SWNT-COOH films. (c) Specific binding tests of thrombin molecules to the SWNT-film aptasensor after treatment with Tween 20. (d) Real-time electrical detection for the specific binding of thrombin molecules with increasing concentrations to the SWNT-film aptasensor. Insets show the sensitivity of the SWNT-film aptasensor with increasing protein concentrations on a log$\log$ scale $(n=4)$.

increased linearly in the protein concentrations from $70 \mathrm{pM}$ to $70 \mathrm{nM}$ on a $\log -\log$ scale $(n=4)$.

\section{Conclusions}

Dielectrophoretically aligned SWNT-films between patterned cantilever electrodes were successfully exploited for the fabrication of aptamer functionalized SWNT-film FET biosensors. The suspended SWNT-film aptasensor exhibited the p-type characteristics in the buffer solution. According to nonspecific binding tests as a control experiment, the aptasensor using SWNT-COOH film without aptamers showed a meaningful current change by the addition of thrombin and VEGF at a concentration of $70 \mathrm{pM}$ with a response time less than $10 \mathrm{~s}$. In addition, after surface treatment with Tween 20, the SWNT-film FET aptasensor demonstrated a real-time, labelfree, step-wise, target-specific, and electrical detection of thrombin molecules down to a concentration of $7 \mathrm{pM}$ with an extraordinary rapid response time of a few seconds and a proportional sensitivity. The novel addressable dielectrophoretically aligned SWNT-film FET aptasensor will be investigated further for various diagnostic applications with biomarker-specific aptamers.

\section{Acknowledgements}

This work was supported by Mid-career Researcher Program through NRF grant funded by the MEST (No. 2009-0085377). This work was supported by the Korea Research Foundation Grant funded by the Korean Government (MOEHRD, Basic Research Promotion Fund, KRF-2008-331-D00753).

\section{Notes and references}

1 M. P. Robertson and A. D. Ellington, Nat. Biotechnol., 2001, 19, 650655.

2 J. Zhang, H. P. Lang, F. Huber, A. Bietsch, W. Grange, U. Certa, R. McKendry, H. J. Guntgerodt, M. Hegner and C. Gerber, Nat. Nanotechnol., 2006, 1, 214-220.

3 H. A. Heus, Nat. Struct. Biol., 1997, 4, 597-600.

4 P. Yin, H. M. T. Choi, C. R. Calvert and N. A. Pierce, Nature, 2008, 451, 318-322. 
5 Y. Xiao, R. Y. Lai and K. W. Plaxco, Nat. Protoc., 2007, 2, 2875 2880.

6 J. Bath and A. J. Turberfield, Nat. Nanotechnol., 2007, 2, 275-284.

7 T. Nguyen, J. P. Hilton and Q. Lin, Microfluid. Nanofluid., 2009, 6, 347-362.

8 H. M. So, K. Won, Y. H. Kim, B. K. Kim, B. H. Ryu, P. S. Na, H. Kim and J. O. Lee, J. Am. Chem. Soc., 2005, 127, 11906-11907.

9 O. S. Kwon, S. J. Park and J. Jang, Biomaterials, 2010, 31, 4740-4747.

10 H. X. Chang, L. H. Tang, Y. Wang, J. H. Jiang and J. H. Li, Anal. Chem., 2010, 82, 2341-2346.

11 H. S. Lee, K. S. Kim, C. J. Kim, S. K. Hahn and M. H. Jo, Biosens. Bioelectron., 2009, 24, 1801-1805.

12 K. S. Kim, H. S. Lee, J. A. Yang, M. H. Jo and S. K. Hahn, Nanotechnology, 2009, 20, 235501.

13 S. N. Kim, J. F. Rusling and F. Papadimitrakopoulos, Adv. Mater., 2007, 19, 3214-3228.

14 S. S. Wong, E. Joselevich, A. T. Woolley, C. L. Cheung and C. M. Lieber, Nature, 1998, 394, 52-55.

15 R. H. Baughman, A. A. Zakhidov and W. A. de Heer, Science, 2002, 297, 787-792.

16 A. Star, E. Tu, J. Niemann, J. C. P. Gabriel, C. S. Joiner and C. Valcke, Proc. Natl. Acad. Sci. U. S. A., 2006, 103, 921-926.
17 H. R. Byon and H. C. Choi, J. Am. Chem. Soc., 2006, 128, 2188-2189.

18 G. A. Zelada-Guillen, J. Riu, A. Duzgun and F. X. Rius, Angew. Chem., Int. Ed., 2009, 48, 7334-7337.

19 D. R. Kim and X. L. Zheng, Nano Lett., 2008, 8, 3233-3237.

20 X. Q. Chen, T. Saito, H. Yamada and K. Matsushige, Appl. Phys. Lett., 2001, 78, 3714-3716.

21 Q. Cao and J. A. Rogers, Adv. Mater., 2009, 21, 29-53.

22 E. S. Snow, J. P. Novak, M. D. Lay and F. K. Perkins, Appl. Phys. Lett., 2004, 85, 4172-4174.

23 M. Lu, M. W. Jang, G. Haugstad, S. A. Campbell and T. H. Cui, Appl. Phys. Lett., 2009, 94, 261903.

24 Heller, A. M. Janssens, J. Mannik, E. D. Minot, S. G. Lemay and C. Dekker, Nano Lett., 2008, 8, 591-595.

25 T. Cang-Rong and G. Pastorin, Nanotechnology, 2009, 20, 255102.

26 Maehashi, T. Katsura, K. Kerman, Y. Takamura, K. Matsumoto and E. Tamiya, Anal. Chem., 2007, 79, 782-787.

27 R. J. Chen, S. Bangsaruntip, K. A. Drouvalakis, N. W. S. Kam, M. Shim, Y. M. Li, W. Kim, P. J. Utz and H. J. Dai, Proc. Natl. Acad. Sci. U. S. A., 2003, 100, 4984- 4989.

28 C. Li, M. Curreli, H. Lin, B. Lei, F. N. Ishikawa, R. Datar, R. J. Cote, M. E. Thompson and C. W. Zhou, J. Am. Chem. Soc., 2005, 127, 12484-12485. 